\title{
New Definitions Will Help, but Cultures are Critical for Resolving Unanswered Questions About Clostridium difficile
}

\author{
Dale N. Gerding, MD
}

There is no question that Clostridium difficile-associated disease (CDAD) rates are on the increase nationally, as shown by US hospital rates. ${ }^{1}$ This observation is confirmed in this issue of the journal by Chandler et al., ${ }^{2}$ specifically for the state of Oregon. As a surrogate measure for increased CDAD rates, I have searched PubMed for publications on C. difficile in this journal and found 26 articles were published in the 5 years from 2001 through 2005, whereas in the previous 10 years only 27 papers had been published-a doubling of the publication rate. With the 9 articles published on C. difficile in this issue of the journal, there should be no question that the CDAD epidemic has now reached the publishing phase, reflecting the increased frequency of CDAD and probably also its increased severity, and that it now rivals methicillin-resistant Staphylococcus aureus (MRSA) infection as the most common healthcare-associated infection in many hospitals.

It is suspected that one reason for the current increase in the incidence of CDAD is the identification of an epidemic strain, variously known as restriction endonuclease analysis (REA) type BI, pulsed-field electrophoresis (PFGE) type NAP1, and polymerase chain reaction (PCR) ribotype 027. This strain has been found in multiple US, Canadian, and European hospitals, ${ }^{3,4}$ including 1 hospital in 2002 in Oregon, where CDAD rates statewide have more than doubled. ${ }^{2}$ Despite knowing that this strain is circulating widely and may be associated with much more severe CDAD than were previous epidemic strains (although this remains to be shown conclusively because of the association of increased patient age with infection caused by the epidemic NAP1 strain), most hospitals, both academic and community, have no idea whether the epidemic strain has reached their institution. ${ }^{5}$ Culture, isolation, and identification of $C$. difficile, once a common procedure in clinical microbiology laboratories in US hospitals, has, with a few exceptions, become almost nonexistent during the past 25 years. As a result, epidemiologic studies are based largely on stool toxin testing, without the benefit of the much more sensitive stool culture results and without the possibility of performing molecular typing and analysis of $C$. difficile isolates to correlate epidemiologic observations with the specific strains of the organism. There has been considerable speculation that the current epidemic strain may be associated with new or different risk factors, yet very few investigators have been able to correlate epidemiologic findings with molecular typing of organisms to determine whether these differences are related to specific strains and/or to other factors.

As we strive to better understand the epidemiology of this largely healthcare-associated infection that may be increasingly common in the community, we are significantly disadvantaged by our current diagnostic methods that rely on toxin testing for diagnosis of disease. Not only are current toxin testing methods insensitive (approximately $40 \%$ of diagnoses are missed by cell cytotoxin testing ${ }^{6}$ or toxin $\mathrm{A}$ immunoassay, ${ }^{7}$ compared with stool culture for toxigenic $C$. difficile), but they also do not result in isolation of the organisms responsible for the disease. Even the widely accepted toxin $\mathrm{A} / \mathrm{B}$ enzyme immunoassay tests are only about $80 \%$ as sensitive as the cell cytotoxin assay, which, in turn, is much less sensitive than culture for toxigenic $C$. difficile. ${ }^{6.8}$ In addition, we often subject the poor patient to the "tyranny of the test result" if physicians believe the falsely negative results and fail to treat the patient. ${ }^{9}$

In this issue of the journal, we see examples of the epidemiologic limitations of our current "cultureless" diagnostic methods. Biller et al., ${ }^{10}$ who worked in collaboration with a superb laboratory at the Centers for Disease Control and Prevention (Atlanta, GA), describe a CDAD outbreak associated initially with moxifloxacin use that did not resolve when the prevailing fluoroquinolone used for treatment was changed to levofloxacin. It is unfortunate that this study was limited to analysis of only $6 \mathrm{C}$. difficile isolates, 3 of which were indistinguishable from the previously identified epidemic NAP1 isolates. If these fluoroquinolone-resistant NAP1 organisms were responsible for this outbreak, it seems highly important to document the proportion of patients with CDAD who were infected with this strain during the 2.5 years

From the Edward Hines, Jr., Veterans Administration Hospital, Hines, Illinois, and the Stritch School of Medicine, Loyola University Chicago, Maywood, Illinois.

Received January 9, 2007; accepted January 9, 2007; electronically published January 31, 2007.

Infect Control Hosp Epidemiol 2007; 28:113-115

(C) 2007 by The Society for Healthcare Epidemiology of America. All rights reserved. 0899-823X/2007/2802-0001\$15.00. 
of this study, and especially during the period of changing fluoroquinolone use. Did isolation of the NAP1 strain increase in frequency? Did the strain disappear from the institution? Did it remain constant in frequency? Unfortunately, we have no idea, but documentation of the frequency of the epidemic strain that was isolated during the course of the study could have resulted in a very different interpretation of the observed fluoroquinolone effects.

Similarly, Lawrence et al. ${ }^{11}$ describe "colonization pressure" as an important risk factor for $\mathrm{CDAD}$ among intensive care unit (ICU) patients but did not document colonization by culture in a single patient, whereas similar previous studies of vancomycin-resistant Enterococcus or MRSA "colonization pressure" obtained samples from patients on a daily to weekly basis to test for the presence of these organisms by culture. ${ }^{12,13}$ A surrogate definition for $C$. difficile colonization based on detection of $C$. difficile toxin in patient's stool (diarrhea was not specified but was implied) was coupled with the arbitrary assumption that such patients were infectious for 14 days and were the only "colonized" patients in the ICU. Even with these approximations of true "colonization pressure," there was a significant association between exposure to these patients and acquisition of CDAD. What remains unknown is how many ICU patients were truly colonized with $C$. difficile and for how long during their ICU stay, not to mention that there was no ability to identify the specific molecular strains or types of $C$. difficile (including nontoxigenic strains) that were acquired by the ICU patients. I am sure the authors would have been delighted to have such information, but were unable to obtain it because of the lack of $C$. difficile isolates for analysis.

In contrast, Barbut et al. ${ }^{14}$ obtained C. difficile isolates routinely in their hospital and performed toxinotyping; PCR ribotyping; and PCR to detect the genes for binary toxin, toxin $A$, and toxin B. As a result, they were able to document a very low rate of transmission of specific $C$. difficile strains in their hospital and to associate the presence of binary toxin genes in the organism with more severe CDAD illness.

The CDAD surveillance definitions recommended by McDonald et al. ${ }^{15}$ add some important missing elements in unifying the epidemiologic definitions for CDAD. The CDAD case definition is commonly accepted, but the definition of severe CDAD has been more variable. If these recommendations are widely accepted (they are currently published without the imprimatur of the Centers for Disease Control and Prevention), they should be of great help in unifying standards of reporting by defining preferred denominators, defining the distinction between healthcare facility-onset and community-onset $\mathrm{CDAD}$, and defining the distinction between healthcare facility-associated and community-associated CDAD. The question of how long after discharge from a healthcare facility a case of community-onset CDAD should be attributed to the healthcare facility may require further tuning as we obtain more information and isolates from this important group of patients. As interest in the incidence and severity of community-associated CDAD increases, these definitions will play an important part in identifying what constitutes a true community-associated case. Again, obtaining C. difficile organisms from such patients becomes ever more important to resolving whether these cases might be caused by $C$. difficile organisms genetically different from those found in healthcare facilities. Finally, as public reporting of CDAD becomes more common, these definitions can serve as a valuable guide.

How can we increase the use of $C$. difficile culture in microbiology laboratories? From the standpoint of treatment, culture and identification of toxigenic C. difficile alone is not a satisfactory approach, because results are obtained too slowly for use in management decisions, and may be obtained too slowly to be any use for rapidly progressive $C$. difficile clinical illness in some patients. One possible solution is to use a stepwise stool testing approach similar to that described by Ticehurst et al. ${ }^{16}$ This method first uses a high-sensitivity rapid screening test, such as the immunoassay for the enzyme glutamate dehydrogenase, to identify stool specimens with negative results, which constitute $80 \%-90 \%$ of all stool specimens submitted to laboratories for $C$. difficile testing. Those specimens with a positive assay result require confirmation of the presence of toxigenic $C$. difficile, because the glutamate dehydrogenase immunoassay lacks specificity. A toxin $A / B$ enzyme immunoassay could be used to rapidly test this small subset of specimens to confirm most positive toxigenic $C$. difficile findings while, at the same time, a stool culture is performed with selective, prereduced, cycloserine-cefoxitinfructose agar media. Isolates obtained from stool specimens that already tested positive by toxin A/B enzyme immunoassay would require no further testing, but isolates from stool specimens that initially tested negative by this assay would have supernatants again tested for toxin $\mathrm{A} / \mathrm{B}$, to screen out nontoxigenic $C$. difficile isolates. The end result would be increased stool-test sensitivity, elimination of most repeated stool testing, and recovery of an isolate from each $C$. difficilepositive stool specimen for purposes of molecular typing and analysis and selective antimicrobial susceptibility testing. Such an approach may be cost-effective and provides the desired increase in test sensitivity and specificity. ${ }^{16}$

Finally, I cannot close without mention of the article by Polgreen et al. ${ }^{17}$ in this issue of the journal, because it illustrates all too clearly the potential unintended consequences of antimicrobial use guidelines and performance measures, whether internally or externally generated. A communityacquired pneumonia guideline was instituted in a small rural hospital but was incorrectly applied to 6 of 12 patients who subsequently developed CDAD. This resulted in unnecessary use of cephalosporin antibiotics to treat these patients, and that in turn led to development of CDAD, which proved to be fatal in 5 of the 15 patients affected. Ironically, inappropriate application of a clinical pathway to improve treatment of community-acquired pneumonia resulted in an unintended increase in the incidence of CDAD. Again, had the 
authors been able to obtain $C$. difficile cultures, I am sure they would have loved to know whether this was a clonal epidemic and whether it was caused by the NAP1 strain. It behooves all of us working in infection control and healthcare epidemiology to work with our respective colleagues in microbiology to develop and implement innovative diagnostic strategies for $C$. difficile infection that will not only be rapid, sensitive, and economical, but will provide isolates of the organism for further analysis. This will enable us to better answer the many pressing epidemiologic questions about $C$. difficile that are before us.

Address reprint requests to Dale N. Gerding, MD, ACOS/Research and Development, Hines VA Hospital, 5th Avenue and Roosevelt Road, Bldg 1, Rm C344, Hines, IL 60141(dale.gerding2@va.gov).

\section{ACKNOWLEDGMENT}

D.N.G. is supported by grants from the US Department of Veterans Affairs Research Service.

\section{REFER E N C ES}

1. McDonald LC, Owings M, Jernigan DB. Clostridium difficile infection in patients discharged from US short-stay hospitals, 1996-2003. Emerg Infect Dis 2006; 12:409-415.

2. Chandler RE, Hedberg K, Cieslak PR. Clostridium difficile-associated Disease in Oregon: increasing incidence and hospital-level risk factors. Infect Control Hosp Epidemiol 2007; 28:116-122 (in this issue).

3. McDonald LC, Killgore GE, Thompson A, et al. An epidemic, toxin gene-variant strain of Clostridium difficile. N Engl J Med 2005; 353: 2433-2441.

4. Loo VG, Poirier L, Miller MA, et al. A predominantly clonal multiinstitutional outbreak of Clostridium-difficile-associated diarrhea with high morbidity and mortality. N Engl J Med 2005; 353:2442-2449.

5. Hubert B, Loo VG, Bourgault A-M, et al. A portrait of the geographic dissemination of the Clostridium difficile North American pulsed-field type 1 strain and epidemiology of C. difficile-associated disease in Quebec. Clin Infect Dis 2007; 44:238-244.
6. Delmee M, Van Broeck J, Simon A, Janssens M, Avesani V. Laboratory diagnosis of Clostridium difficile-associated diarrhoea: a plea for culture. J Med Microbiol 2005; 54:187-191.

7. Shanholtzer CJ, Willard KE, Holter JJ, Olson MM, Gerding DN, Peterson LR. Comparison of the VIDAS Clostridium difficile toxin A immunoassay with C. difficile culture and cytotoxin and Latex tests. J Clin Microbiol 1992; 30:1837-1840.

8. O'Connor D, Hynes P, Cormican M, Collins E, Corbett-Feeney G, Cassidy M. Evaluation of methods for detection of toxins in specimens of feces submitted for diagnosis of Clostridium difficile-associated diarrhea. J Clin Microbiol 2001; 39:2846-2849.

9. Johnson S, Kent SA, O'Leary KJ, et al. Fatal pseudomembranous colitis associated with a variant Clostridium difficile strain not detected by toxin A immunoassay. Ann Intern Med 2001; 135:434-438.

10. Biller $\mathrm{P}$, Shank B, Lind $\mathrm{L}$, et al. Moxifloxacin therapy as a risk factor for Clostridium difficile-associated disease during an outbreak: attempts to control a new epidemic strain. Infect Control Hosp Epidemiol 2007; 28: 198-201 (in this issue).

11. Lawrence SJ, Puzniak LA, Shadel BN, Gillespie KN, Kollef MH, Mundy LM. Clostridium difficile in the intensive care unit: epidemiology, costs, and colonization pressure. Infect Control Hosp Epidemiol 2007; 28: 123-130 (in this issue).

12. Bonten MJ, Slaughter S, Ambergen AW, et al. The role of "colonization pressure" in the spread of vancomycin-resistant enterococci: an important infection control variable. Arch Intern Med 1998; 158:1127-1132.

13. Merrer J, Santoli F, Appere-De Vecchi C, Tran B, De Jonghe B, Outin H. "Colonization pressure" and risk of acquisition of methicillin-resistant Staphylococcus aureus in a medical intensive care unit. Infect Control Hosp Epidemiol 2000; 21:718-723.

14. Barbut F, Gariazzo B, Bonnet L, et al. Clinical features of Clostridium difficile-associated infections and molecular characterization of strains: results of a retrospective study, 2000-2004. Infect Control Hosp Epidemiol 2007; 28:131-139 (in this issue).

15. McDonald LC, Coignard B, Dubberke E, et al. Recommendations for surveillance of Clostridium difficile-associated disease. Infect Control Hosp Epidemiol 2007; 28:140-145 (in this issue).

16. Ticehurst JR, Aird DZ, Dam LM, Borek AP, Hargrove JT, Carroll KC. Effective detection of toxigenic Clostridium difficile by a two-step algorithm including tests for antigen and cytotoxin. J Clin Microbiol 2006; 44:1145-1149.

17. Polgreen PM, Chen YY, Cavanaugh JE, et al. An outbreak of severe Clostridium difficile-associated disease possibly related to inappropriate antimicrobial therapy for community-acquired pneumonia. Infect Control Hosp Epidemiol 2007; 28:212-214 (in this issue). 